\title{
The New Entity of Paranodopathies: A Target Structure with Therapeutic Consequences
}

\author{
(ㄷ)(우(ㅇ) \\ Authors \\ Kathrin Doppler, Claudia Sommer \\ Affiliation \\ Neurological Clinic, University Hospital Würzburg \\ Key words \\ neuropathy, node of Ranvier, autoantibody, CIDP \\ Bibliography \\ DOI http://dx.doi.org/10.1055/s-0043-102455 \\ Neurology International Open 2017; 1: E56-E60 \\ (c) Georg Thieme Verlag KG Stuttgart · New York \\ ISSN 2511-1795 \\ Correspondence \\ Dr. Kathrin Doppler \\ Neurological Clinic \\ University Hospital Würzburg \\ Josef-Schneider-Str. 11 \\ 97080 Würzburg \\ Doppler_K@ukw.de
}

\begin{abstract}
Autoimmune neuropathies with autoantibodies against paranodal proteins have been described in the last few years. They comprise a subgroup of inflammatory neuropathies with $\lg \mathrm{G} 4$ autoantibodies against the paranodal proteins neurofascin-155, contactin-1 and caspr-1. Although this subtype of autoimmune neuropathy represents less than $10 \%$ of all patients diagnosed with CIDP, it is of high interest as they show a different response to treatment. Even though there are no therapeutic studies available due to the limited number of patients identified so far, all case reports published so far report an excellent response to treatment with rituximab, and in most cases no response to treatment with IVIG, the standard therapy of CIDP. The typical clinical picture of patients with autoantibodies against paranodal proteins is characterized by an acute onset of a severe predominantly motor neuropathy, often accompanied by action tremor and sensory ataxia, with demyelinating features in the nerve conduction studies but an axonal histological phenotype. The latter may be explained by the pathogenetic concept of a paranodopathy/nodopathy, a disease of the paranode/node of Ranvier.
\end{abstract}

Inflammatory polyneuropathies are present in clinical practice as a heterogeneous group of diseases, not only in terms of clinical symptoms, but also in terms of their therapeutic response. Reliable biomarkers are lacking both for diagnostics and as markers of response to specific therapies. This is primarily due to the fact that the pathogenesis of the immunneuropathies is still largely unexplained. $T$ cell-mediated, auto-antibody-induced, complement-mediated damage mechanisms as well as a damaged blood-nerve barrier are discussed as possible causes of autoimmune-inflammatory neuropathies [1]. Therefore, immuno-neuropathies, in particular the chronic inflammatory demyelinating polyradiculoneuropathy (CIDP), are not only clinically but also pathophysiologically a heterogeneous disease group. A subform of the CIDP are e. g., ganglioside autoantibody neuropathies, which account for only a few percent of the CIDP cases but, depending on the antigen, exhibit characteristic disease patterns [2]. In recent years, a further subform of autoantibody-associated neuropathies has been identified: immuno-neuropathies with autoantibodies against paranodal proteins [3].

\section{The node of Ranvier - more than just a gap in the Myelin Sheath}

Paranodium is the portion of the nerve fiber directly adjoining the sides of the node of Ranvier. It demarcates the node, on which the sodium channels important for the saltatorial excitation are localized, from the juxtaparanode, where potassium channels are located [4]. The myelin sheath is connected to the axon via a complex of the paranodal proteins neurofascin-155, contactin-1 and caspr1 ( $\triangleright$ Fig. 1). This complex serves, for example, as diffusion barrier for ion channels [5]. It has been described in various studies [6-10] that especially in the case of demyelinating neuropathies, changes in the node of Ranvier architecture can occur. It has been known for a long time that, in the case of primarily demyelinating polyneuropathies, segmental demyelination begins with an elongation of the node and extends in the direction of internodes, and a decrease in node length occurs again during remyelination [11-13]. Recently, however, proteins of the node of Ranvier have been identified as antigens for inflammatory neuropathies [14-18].

\section{Neurofascin-155 Autoantibodies}

Autoantibodies to neurofascin-155 were first described in multiple sclerosis, since neurofascin-155 is also present in paranodes of the CNS [19]. The extent to which these contribute to the pathogenesis of multiple sclerosis as part of the humoral immune response has not yet been finally clarified. In 2012, $\mathrm{Ng}$ et al. showed the presence of autoantibodies against neurofascin-155 (the paranodal isoform) and -186 (the nodally localized isoform) in $4 \%$ of patients with Guillain-Barré syndrome and CIDP using ELISA and cell-based assay [17]. 
Although the 2 isoforms differ only slightly, isotype-specific autoantibodies are present in almost all patients [17]. A uniform clinical phenotype, consisting of severe motor symptoms, acute onset, poor response to intravenous immunoglobulins (IVIG) and cerebellar tremor, was reported by Querol et al. [20]. This clinical phenotype was confirmed in follow-up studies [21,22]. Neurofascin-155 autoantibodies have also been described in patients with CIDP in combination with a central involvement, but there are contradictory findings with regard to a preferential occurrence of neurofascin-155 autoantibodies in this subgroup [23, 24]. The prevalence of neurofascin-155 autoantibodies in patients with CIDP was approximately $4 \%$ [20] in a European cohort, $3 \%$ in a cohort with Japanese and European patients, and $7 \%$ in Japanese cohorts [21] and $18 \%$ [22]. The extent to which ethnic differences and a different sensitivity and specificity of the different assays play a role currently cannot be determined with certainty. However, it can be assumed that neurofascin-155 autoantibodies occur in a subgroup of patients diagnosed with a CIDP and are associated with a characteristic phenotype.

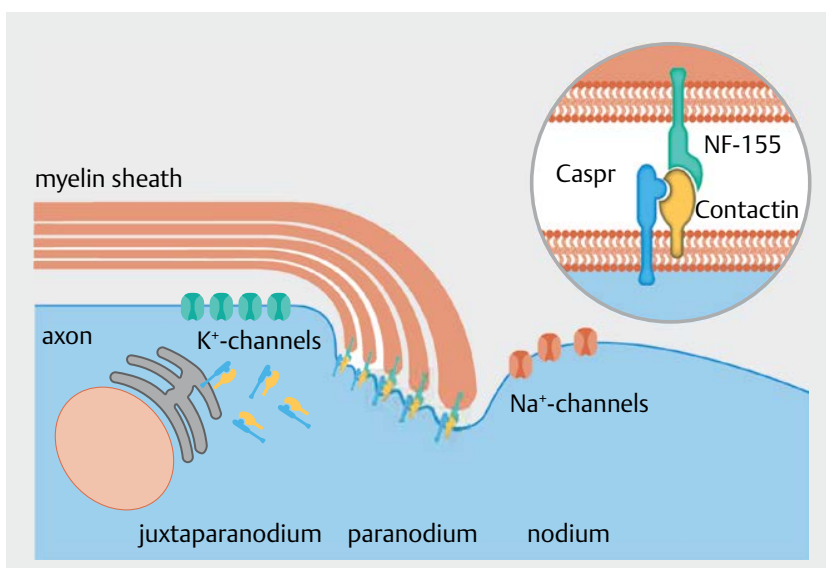

- Fig. 1 Molecular structure of the node of Ranvier. The node of Ranvier is divided into the section juxtaparanode (potassium channels), paranode (paranodal protein complex) and node (sodium channels). The paranodal protein complex consists of the proteins Caspr, neurofascin-155 (NF-155) and contactin and connects the myelin sheath to the axon [40].

\section{Contactin-1 Autoantibody}

In 2013, Querol et al. detected autoantibodies to contactin-1 in 2 patients with CIDP, and autoantibodies to the protein complex of contactin-1 and Caspr in a further patient [16]. Clinically, the patients were characterized by acute onset severe polyneuropathy, predominantly motor in character. A study carried out in our clinic found that in 3 out of 4 patients identified with contactin- 1 autoantibodies also exhibited intention tremor [25] whereas in a Japanese cohort, sensory ataxia was clinically important [26]. The prevalence in the cohorts studied so far is $2.4-7.5 \%[16,25,26]$.

\section{Caspr-1 Autoantibody}

We recently identified in our cohort of patients with inflammatory neuropathies autoantibodies to the third paranodal protein Caspr1 [27]. While neurofascin- 155 and contactin- 1 autoantibodies were almost exclusively detectable in patients with chronic inflammatory polyneuropathy, Caspr-1 autoantibodies were detected in a patient with Guillain-Barré syndrome and a patient with CIDP. Both exhibited severe, acutely onset polyneuropathy, predominantly motor, and with pronounced neuropathic pain. The prevalence in our cohort was $3.5 \%$, but both prevalence and clinical phenotype must be confirmed in further studies ( $\vee$ Table $\mathbf{1}$ ).

\section{Assays for the detection of autoantibodies to paranodal proteins}

So far, various assays have been used for the detection of autoantibodies to paranodal proteins. The ELISA was predominantly used as a screening instrument $[17,25,26]$. However, the selection of the protein has to be taken into account: For neurofascin-155 it has been shown in several studies that the use of neurofascin-155-NS0 of the rat can result in a nonspecific binding $[17,28,29]$. Therefore, coating the ELISA plates with human neurofascin-155 is recommended. Another frequently used test is the binding experiment with HEK293 cells transfected with the DNA of the respective antigen, also in combination with flow cytometry for quantification $[16,17,27]$. For detection of the paranodal binding, binding assays were carried out with murine teased fibers in almost all studies $[16,20,25,27]$. Thus, although with these experiments the specific antigen cannot be determined, a paranodal binding can be demonstrated. Western blot was only used in a few studies and showed false-negative results in individual patients, possibly due

- Table 1 Overview of the characteristics of each autoantibody against paranodal proteins.

\begin{tabular}{|c|c|c|c|}
\hline & Neurofascin-155 & Contactin-1 & Caspr-1 \\
\hline Prevalence in CIDP & $3-18 \%$ & $2.4-7.5 \%$ & са. $3 \%$ \\
\hline Literature & $\begin{array}{l}\mathrm{Ng} \text { et al., 2012, Kawamura et al., 2013, Querol } \\
\text { et al., 2014, Ogata et al., 2015, Devaux et al., } \\
2016\end{array}$ & $\begin{array}{l}\text { Querol et al., 2013, Doppler et al., } \\
\text { 2015, Miura et al., } 2015\end{array}$ & Doppler et al., 2016 \\
\hline \multirow[t]{3}{*}{ Clinical phenotype } & Acute onset & Acute onset & Acute onset \\
\hline & Motor > sensory & Motor > sensory & Motor > sensory \\
\hline & Cerebellar tremor & (Intention tremor) & Neuropathic pain \\
\hline IgG-Subclasses & $\lg G 4>\lg G 3>\lg G 1$ & $\lg G 4>\operatorname{lgg} 3$ & $\lg G 4$ \\
\hline
\end{tabular}



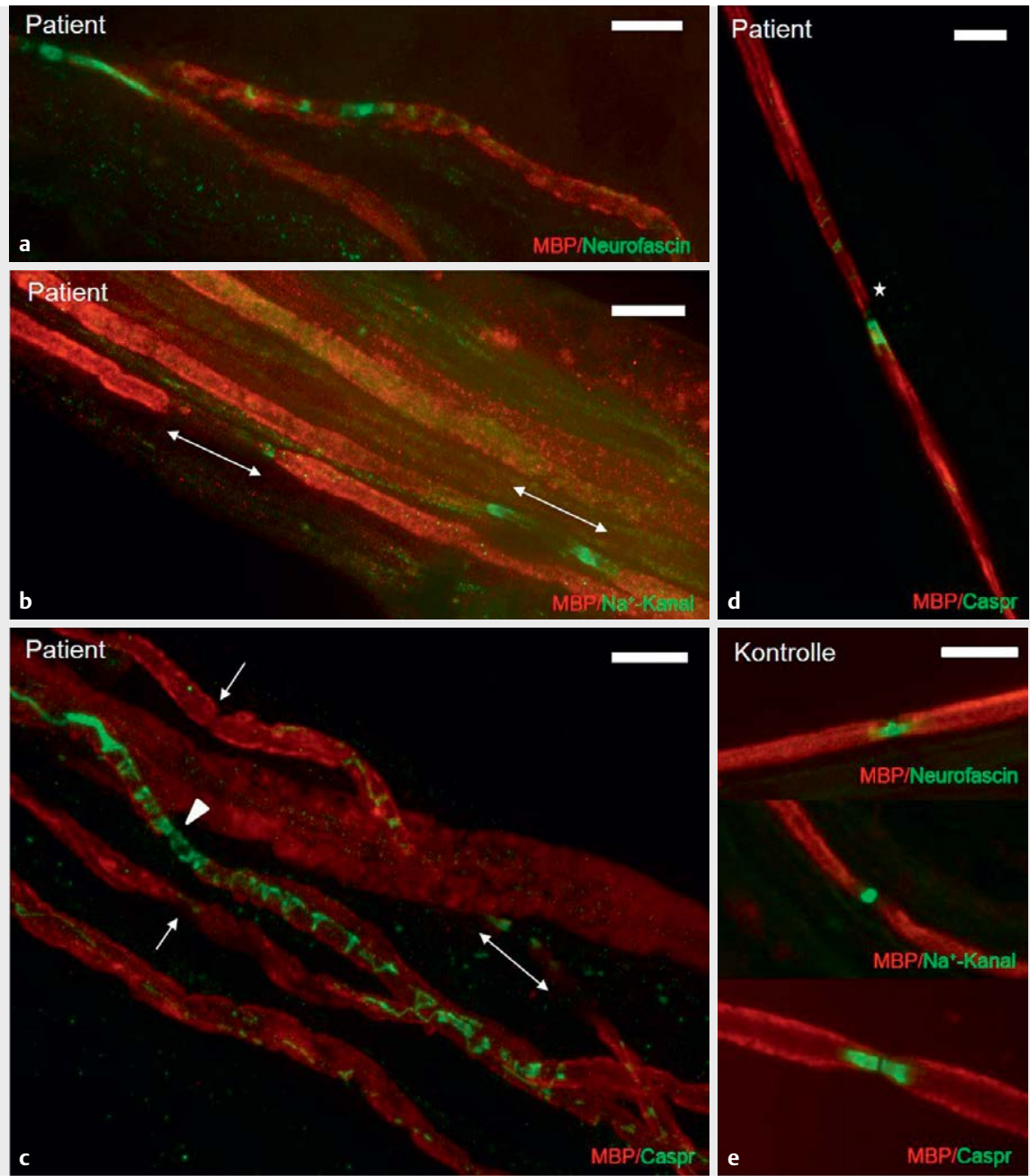

Fig. 2 Destruction of the node of Ranvier architecture in the teased fiber of sural nerve biopsy of a patient with autoantibodies to the paranodal protein Caspr, fluorescence double staining with myelin basic protein (MBP, myelin marker, red) and anti-Caspr c, d, anti-neurofascin a and Anti-sodium channel $\mathbf{b}$ (in each case green), in $\mathbf{e}$ in comparison the staining of a control nerve. There is a misdistribution of neurofascin $\mathbf{a}$ and the sodium channels $\mathbf{b}$. The double arrows in $\mathbf{b}, \mathbf{c}$ mark elongated nodes. Some myelin sheath gaps show an almost complete loss of Caspr immunoreactivity (c, arrows), partly also on one side (d, asterisks). Other fibers show a distribution of Caspr into the juxtaparanode and internode $\mathbf{c}$ [27]. Bars 10 $\mu \mathrm{m}$.

to the denaturation of the protein $[25,29]$. In summary, the ELISA appears to be a reliable screening tool, and binding experiments with transfected HEK293 cells, or directly with the nerve tissue, are suitable as confirmatory tests.

\section{Pathophysiology: Concept of Paranodopathy}

Although neuropathies with autoantibodies to paranodal proteins are classified as subtypes of CIDP, it is not a classical demyelinating polyneuropathy. In contrast to the CIDP, in nerve biopsies, there are no signs of demyelination/remyelination, such as onion bulb formations or thinly myelinated fibers $(22,25,27)$. This is because the myelin sheath is not the point of attack of the autoantibodies. Electrophysiologically, there are conduction blocks, prolonged F-latencies and distal motor latencies, and reduced nerve conduction velocity, so that the electrophysiological criteria of a CIDP are usu- ally met $[16,20,25,27]$. This apparent discrepancy between histology and electrophysiology is explained by the concept of paranodopathy/nodopathy, a disease targeting the complex nodal region [30,31]. The hypothesis is that the autoantibodies interfere with the adhesion of the paranodal proteins and thus interfere with the connection between axon and myelin sheath, which could lead to elongation of the nerve and dispersion of ion channels at the node of Ranvier of the elongated nerve. Thus, the presence of conduction blocks and the extended distal motor latency can be explained by the lengthening of the myelin sheath gaps as well as the reduced nerve conduction velocity [31]. In fact, it was shown in vitro that autoantibodies to contactin- 1 inhibit the adhesion of the paranodal proteins and lead to the destruction of their architecture [32]. The latter was confirmed in skin nerves and nerve teased nerve fibers of patients with contactin-1, Caspr-1, and neurofascin-155-associated neuropathy [22, 25, 27] (• Fig. 2). 


\section{Role of IgG subclasses}

In the majority of cases, autoantibodies to paranodal proteins are immunoglobulins of subclass IgG4 [17, 20, 25-27]. This has a special role among the immunoglobulins in that it does not activate complement and is predominantly functionally monovalent and thus does not lead to cross-linking of antigens [33, 34]. Therefore, in the majority of patients, one cannot assume the presence of a complement-mediated inflammatory reaction, which is pathogenically relevant in neuropathies with ganglioside autoantibodies. A possible pathophysiological mechanism, however, is antibody binding-induced transformational processes in the node of Ranvier, as described above. It is assumed that the lack of efficacy for IVIG in these patients is associated with the lack of complement activation [20]. It is known that IVIG inhibits complement activation even if the exact mode of IVIG action is still not elucidated in detail [35, 36]. However, not all autoantibodies to paranodal proteins belong to the IgG4 subclass. Patients with contactin- 1 and Caspr autoantibodies with lgG3 predominance have also been described. Also, most patients with predominant IgG4 autoantibodies are by no means exclusively IgG4. Thus, it was shown that sera from patients with predominant IgG4 lead to a complement binding, presumably by $\lg G 3$, though not abundantly present [37]. The extent to which IgG3 autoantibodies are pathogenic remains to be clarified in vivo. For IgG4 autoantibodies to contactin-1, a potential pathogenicity has already been demonstrated in the passive transfer model, but not yet for $\operatorname{lgG} 3$ [38]. The cause for the presence of different $\lg G$ subclasses with the same antigen is not yet known.

\section{Therapy of neuropathy with antibodies to paranodal antibodies}

The number of patients known to have autoantibodies to paranodal proteins is just as small as the number of studies. It is not surprising that there are no studies as yet on therapeutic strategies. However, in the available case reports, impressive and unifrom response to rituximab of patients with IgG4 autoantibodies against neurofascin-155, contactin-1 and Caspr is described [25, 27, 39]. A temporary response to corticosteroids and plasmapheresis has also also been reported, and occasionally in patients with IgG3 autoantibodies also response to IVIG [25, 27, 37]. However, the clearest and most sustained improvement is seen after administration of rituximab, so that in patients with IgG4 autoantibodies to paranodal antigens, contrary to the guidelines for CIDP therapy, this can well be the first choice drug. The extent to which this is also true of the rare occurrence of predominantly lgG3 autoantibodies to paranodal proteins cannot as yet be estimated. In patients with relatively acute onset of predominantly motor inflammatory neuropathy, the presence of autoantibodies to paranodal proteins should be suspected, especially in the case of poor response to IVIG. If there is evidence for the presence of autoantibody, given available evidence, we consider initiation of therapy with rituximab as reasonable.

Commercial tests for the detection of autoantibodies to paranodal proteins are not currently available, but research labs (for example, Würzburg University Hospital, Neurology) can help demonstrate the presence of these antibodies.

\section{Conclusion}

Neuropathies with autoantibodies to paranodal proteins are a new subgroup of immune neuropathies. The characteristic clinical picture is a relatively acute onset of severe motor neuropathy, partly together with cerebellar tremor. The autoantibodies predominantly belong to the subclass IgG4 and patients typically respond well to therapy with rituximab but most often not to IVIG.

\section{Conflict of interest}

The authors declare no conflict of interest.

\section{References}

[1] Mathey EK, Park SB, Hughes RA et al. Chronic inflammatory demyelinating polyradiculoneuropathy: from pathology to phenotype. J Neurol Neurosurg Psychiatry 2015; 86: 973-985

[2] Quarles RH, Weiss MD. Autoantibodies associated with peripheral neuropathy. Muscle Nerve 1999; 22: 800-822

[3] Querol L, Illa I. Paranodal and other autoantibodies in chronic inflammatory neuropathies. Curr Opin Neurol 2015; 28: 474-479

[4] Salzer JL, Brophy PJ, Peles E. Molecular domains of myelinated axons in the peripheral nervous system. Glia 2008; 56: 1532-1540

[5] Rosenbluth J. Multiple functions of the paranodal junction of myelinated nerve fibers. J Neurosci Res 2009; 87: 3250-3258

[6] Doppler K, Werner C, Sommer C. Disruption of nodal architecture in skin biopsies of patients with demyelinating neuropathies. J Peripher Nerv Syst 2013; 18: 168-176

[7] Doppler K, Werner C, Henneges C et al. Analysis of myelinated fibers in human skin biopsies of patients with neuropathies. J Neurol 2012; 259: $1879-1887$

[8] Li J, Bai Y, Ghandour K et al. Skin biopsies in myelin-related neuropathies: bringing molecular pathology to the bedside. Brain 2005; 128 : 1168-1177

[9] Cifuentes-Diaz C, Dubourg O, Irinopoulou T et al. Nodes of ranvier and paranodes in chronic acquired neuropathies. PLoS One 2011; 6: e14533

[10] Peltier AC, Myers MI, Artibee KJ et al. Evaluation of dermal myelinated nerve fibers in diabetes mellitus. J Peripher Nerv Syst 2013; 18: 162-167

[11] Allt G, Cavanagh JB. Ultrastructural changes in the region of the node of ranvier in the rat caused by diphtheria toxin. Brain 1969; 92 : 459-468

[12] Allt G. Repair of segmental dehyelination in peripheral nerves: an electron microscope study. Brain 1969; 92: 639-646

[13] Weller RO, Nester B. Early changes at the node of Ranvier in segmental demyelination. Histochemical and electron microscopic observations. Brain 1972; 95: 665-674

[14] Devaux JJ, Odaka M, Yuki N. Nodal proteins are target antigens in Guillain-Barre syndrome. J Peripher Nerv Syst 2012; 17: 62-71

[15] Devaux JJ. Antibodies to gliomedin cause peripheral demyelinating neuropathy and the dismantling of the nodes of Ranvier. Am J Pathol 2012; 181: 1402-1413

[16] Querol L, Nogales-Gadea G, Rojas-Garcia R et al. Antibodies to contactin-1 in chronic inflammatory demyelinating polyneuropathy. Ann Neurol 2013; 73: 370-380 
[17] $\mathrm{Ng}$ JK, Malotka J, Kawakami N et al. Neurofascin as a target for autoantibodies in peripheral neuropathies. Neurology 2012; 79: 2241-2248

[18] Pruss H, Schwab JM, Derst C et al. Neurofascin as target of autoantibodies in Guillain-Barre syndrome. Brain 2011; 134: e173

[19] Mathey EK, Derfuss T, Storch MK et al. Neurofascin as a novel target for autoantibody-mediated axonal injury. J Exp Med 2007; 204: 23632372

[20] Querol L, Nogales-Gadea G, Rojas-Garcia R et al. Neurofascin IgG4 antibodies in CIDP associate with disabling tremor and poor response to IVIg. Neurology 2014; 82: 879-886

[21] Devaux J], Miura Y, Fukami Y et al. Neurofascin-155 lgG4 in chronic inflammatory demyelinating polyneuropathy. Neurology 2016; 86: 800-807

[22] Ogata H, Yamasaki R, Hiwatashi A et al. Characterization of IgG4 anti-neurofascin 155 antibody-positive polyneuropathy. Ann Clin Transl Neurol 2015; 2: 960-971

[23] Kawamura N, Yamasaki R, Yonekawa T et al. Anti-neurofascin antibody in patients with combined central and peripheral demyelination. Neurology 2013; 81: 714-722

[24] Vural A, Gocmen R, Kurne AT et al. Fulminant central plus peripheral nervous system demyelination without antibodies to neurofascin. Can J Neurol Sci 2016; 43: 149-156

[25] Doppler K, Appeltshauser L, Wilhelmi K et al. Destruction of paranodal architecture in inflammatory neuropathy with anti-contactin-1 autoantibodies. J Neurol Neurosurg Psychiatry 2015; 86: 720-728

[26] Miura Y, Devaux J], Fukami Y et al. Contactin $1 \mathrm{lgG} 4$ associates to chronic inflammatory demyelinating polyneuropathy with sensory ataxia. Brain 2015; 138: 1484-1491

[27] Doppler K, Appeltshauser L, Villmann C et al. Auto-antibodies to contactin-associated protein 1 (Caspr) in two patients with painful inflammatory neuropathy. Brain 2016; 139: 2617-2630

[28] Cortese A, Devaux J], Zardini E et al. Neurofascin-155 as a putative antigen in combined central and peripheral demyelination. Neurol Neuroimmunol Neuroinflamm 2016; 3: e238

[29] Kadoya M, Kaida K, Koike H et al. IgG4 anti-neurofascin155 antibodies in chronic inflammatory demyelinating polyradiculoneuropathy: Clinical significance and diagnostic utility of a conventional assay. J Neuroimmunol 2016; 301: 16-22
[30] Uncini A. A common mechanism and a new categorization for anti-ganglioside antibody-mediated neuropathies. Exp Neurol 2012; 235: 513-516

[31] Uncini A, Susuki K, Yuki N. Nodo-paranodopathy: beyond the demyelinating and axonal classification in anti-ganglioside antibody-mediated neuropathies. Clin Neurophysiol 2013; 124: 1928-1934

[32] Labasque M, Hivert B, Nogales-Gadea G et al. Specific contactin $\mathrm{N}$-glycans are implicated in neurofascin binding and autoimmune targeting in peripheral neuropathies. J Biol Chem 2014; 289: 7907-7918

[33] Tao MH, Smith RI, Morrison SL. Structural features of human immunoglobulin $\mathrm{G}$ that determine isotype-specific differences in complement activation. J Exp Med 1993; 178: 661-667

[34] Vidarsson G, Dekkers G, Rispens T. IgG subclasses and allotypes: from structure to effector functions. Front Immunol 2014; 5: 520

[35] Yuki N, Watanabe H, Nakajima T et al. IVIG blocks complement deposition mediated by anti-GM1 antibodies in multifocal motor neuropathy. J Neurol Neurosurg Psychiatry 2011; 82: 87-91

[36] Piepers S, Jansen MD, Cats EA et al. IVIg inhibits classical pathway activity and anti-GM1 IgM-mediated complement deposition in MMN. J Neuroimmunol 2010; 229: 256-262

[37] Appeltshauser L, Weishaupt A, Sommer C et al. Complement deposition induced by binding of anti-contactin-1 auto-antibodies is modified by immunoglobulins. Exp Neurol 2016

[38] Manso C, Querol L, Mekaouche M et al. Contactin-1 IgG4 antibodies cause paranode dismantling and conduction defects. Brain 2016

[39] Querol L, Rojas-Garcia R, Diaz-Manera J et al. Rituximab in treatment-resistant CIDP with antibodies against paranodal proteins. Neurol Neuroimmunol Neuroinflamm 2015; 2: e149

[40] Boyle ME, Berglund EO, Murai KK et al. Contactin orchestrates assembly of the septate-like junctions at the paranode in myelinated peripheral nerve. Neuron 2001; 30: 385-397 